\title{
SYSTEMS OF INEQUALITIES INVOLVING CONVEX FUNCTIONS ${ }^{1}$
}

\author{
KY FAN, IRVING GLICKSBERG, AND A. J. HOFFMAN
}

1. Let $X$ be a vector space ${ }^{2}$ over the real field, and let $K$ be a nonempty convex set in $X$. As usual, a real-valued function $f$ defined on $K$ is said to be convex, if $f\left(\alpha_{1} x_{1}+\alpha_{2} x_{2}\right) \leqq \alpha_{1} f\left(x_{1}\right)+\alpha_{2} f\left(x_{2}\right)$ holds for any two elements $x_{1}, x_{2} \in K$ and for any $\alpha_{1} \geqq 0, \alpha_{2} \geqq 0, \alpha_{1}+\alpha_{2}=1 . f$ is said to be concave, if $-f$ is convex.

Let $f_{i}(1 \leqq i \leqq m)$ be $m$ convex functions defined on $K$. The system of inequalities

$$
f_{i}(x)<0
$$$$
(1 \leqq i \leqq m)
$$

is said to be consistent, if an element $x \in K$ satisfying all $m$ inequalities (i.e. a solution of (1)) does exist. Otherwise (1) is said to be inconsistent. A system (1) is said to be irreducibly inconsistent, if it is inconsistent and if every proper subsystem of (1) is consistent.

The convex functions $f_{1}, f_{2}, \cdots, f_{m}$ are said to be linearly independent, if no linear combination $\sum_{i=1}^{m} \lambda_{i} f_{i}$ with real coefficients $\lambda_{i}$, not all zero, can remain $\geqq 0$ throughout $K$. In the special case when $K=X$ and when the $f_{i}$ 's are linear forms on $X$, this definition of linear independence agrees with the usual one. Indeed, a linear form can remain $\geqq 0$ throughout the entire vector space $X$ only when it is identically zero. Incidentally. we observe that there exist arbitrarily many linearly independent convex functions even on a one-dimensional convex set. For example, the $m$ convex functions $f_{i}(x)$ $=x^{i}-1 /(i+1)(1 \leqq i \leqq m)$ on the unit interval $0 \leqq x \leqq 1$ are linearly independent for any natural number $m$. In fact, if some $m$ real numbers $\lambda_{i}$ satisfy the relation $\sum_{i=1}^{m} \lambda_{i}\left(x^{i}-1 /(i+1)\right) \geqq 0$ for $0 \leqq x \leqq 1$, then since we have $\int_{0}^{1}\left[\sum_{i=1}^{m} \lambda_{i}\left(x^{i}-1 /(i+1)\right)\right] d x=0$, the polynomial $\sum_{i=1}^{m} \lambda_{i}\left(x^{i}-1 /(i+1)\right)$ must vanish identically and therefore all $\lambda_{i}=0$.

The purpose of this note is to prove the following results concerning a system (1) of inequalities with convex functions $f_{i}$ defined on $K$.

Received by the editors April 24, 1956 and, in revised form, August 10, 1956 and September 17, 1956.

1 The work of the first author was prepared in part at the University of Notre Dame, on a project supported by a grant from the National Science Foundation; and in part under a National Bureau of Standards contract with The American University. The work of the third author was sponsored in part by the Office of Naval Research.

${ }^{2}$ Notice that $X$ is not topologized, and that the dimension of $X$ is arbitrary, finite or infinite. 
THEOREM 1. Either the system (1) is consistent, or there exist m numbers $\lambda_{i} \geqq 0$, not all zero, such that

$$
\sum_{i=1}^{m} \lambda_{i} f_{i}(x) \geqq 0 \quad \text { for all } x \in K .
$$

The two alternatives exclude each other.

THEOREM 2. Let the system (1) be consistent, and let $g$ be a concave function defined on $K$. Then:

(i) The inequality

$$
g(x) \leqq 0
$$

is a conseguence of (1) [i.e., every $x \in K$ satisfying (1) also satisfies (3) ], if and only if there exist $m$ numbers $\lambda_{i} \geqq 0$ such that

$$
g(x) \leqq \sum_{i=1}^{m} \lambda_{i} f_{i}(x) \quad \text { for all } x \in K .
$$

(ii) The supremum $\gamma$ of $g(x)$, when $x \in K$ varies over all solutions of (1), is finite, if and only if there exist $m$ numbers $\lambda_{i} \geqq 0$ such that $g(x)-\sum_{i=1}^{m} \lambda_{i} f_{i}(x)$ is bounded above on $K$. When this condition is satisfied, we have

$$
\gamma=\operatorname{Min}_{\lambda_{i} \geq_{0}} \operatorname{Sup}_{x \in K}\left\{g(x)-\sum_{i=1}^{m} \lambda_{i} f_{i}(x)\right\} .
$$

THEOREM 3. Let the system (1) be consistent, and let $g$ be a concave function defined on $K$. If inequality (3) is a consequence of the syitem (1), and if (3) is not a consequence of any proper subsystem of (1), then the convex functions $f_{1}, f_{2}, \cdots, f_{m}$ are linearly independent.

THEOREM 4. The system (1) is irreducibly inconsistent, if and only if the following two conditions are both satisfied: (a) Any $m-1$ of the convex functions $f_{1}, f_{2}, \cdots, f_{m}$ are linearly independent; (b) there exist $m$ positive numbers $\lambda_{i}$ satisfying (2).

2. Before proving these theorems, we shall discuss their relationship to certain known results.

First, if $X$ is a finite dimensional real vector space, $K$ is the convex set formed by all vectors with positive components, and if the $f_{i}$ 's are linear forms, then Theorem 1 becomes von Neumann's theorem of the alternative for matrices [6, pp. 138-143].

Next, consider a system of linear inequalities

$$
\phi_{i}(x)>\alpha_{i}, \quad(1 \leqq i \leqq m)
$$


where $\phi_{i}$ are linear forms on a real vector space $X$, and $\alpha_{i}$ are real numbers. Applying Theorem 1 to $K=X, f_{i}=\alpha_{i}-\phi_{i}$, then the system (6) is consistent on $X$, if and only if

$$
\sum_{i=1}^{m} \lambda_{i} \alpha_{i}<\operatorname{Sup}_{x \in X} \sum_{i=1}^{m} \lambda_{i} \phi_{i}(x)
$$

holds for any $m$ numbers $\lambda_{i} \geqq 0$ which are not all zero. But $\sum_{i=1}^{m} \lambda_{i} \phi_{i}$ being a linear form on $X$, the supremum on the right hand side of (7) is $+\infty$ except when $\sum_{i=1}^{m} \lambda_{i} \phi_{i}=0$. Hence (6) is consistent on $X$, if and only if, for any $m$ numbers $\lambda_{i} \geqq 0$, not all zero, the relation $\sum_{i=1}^{m} \lambda_{i} \phi_{i}=0$ implies $\sum_{i=1}^{m} \lambda_{i} \alpha_{i}<0$. This is a theorem due to W. B. Carver [2, Theorem 3].

Similarly, Theorem 4 includes the following result of Carver [2, Theorem 2]: The system of linear inequalities (6) is irreducibly inconsistent on $X$, if and only if any $m-1$ of the linear forms $\phi_{i}$ are linearly independent and there exist $m$ positive numbers $\lambda_{i}$ such that $\sum_{i=1}^{m} \lambda_{i} \phi_{i}=0, \sum_{i=1}^{m} \lambda_{i} \alpha_{i} \geqq 0$.

Assume now that the system (6) is consistent on $X$. Let $\psi$ be a linear form on $X$ and $\beta$ a real number. By Theorem 2, (i), the inequality $\psi(x) \geqq \beta$ is a consequence of (6) on $X$, if and only if there exist $m$ numbers $\lambda_{i} \geqq 0$ such that

$$
\beta-\psi(x) \leqq \sum_{i=1}^{m} \lambda_{i}\left[\alpha_{i}-\phi_{i}(x)\right] \quad \text { for all } x \in X .
$$

Since $\psi-\sum_{i=1}^{m} \lambda_{i} \phi_{i}$ is a linear form, the truth of the last inequality for all $x \in X$ is equivalent to

$$
\psi=\sum_{i=1}^{m} \lambda_{i} \phi_{i} \text { and } \beta \leqq \sum_{i=1}^{m} \lambda_{i} \alpha_{i} .
$$

Thus we are led to a result which is analogous to a classical theorem of J. Farkas [4, pp. 5-7] and H. Weyl [7, Satz 3] concerning a system of linear inequalities $\phi_{i}(x) \geqq \alpha_{i}$ [instead of the strict inequalities (6)]. Similarly, Theorem 2, (ii) includes the following result: The infimum of $\psi(x)$, when $x$ varies over all solutions of the consistent system (6) on $X$, is finite, if and only if $\psi$ is a linear combination of the $\phi_{i}$ 's with non-negative coefficients. When this condition is satisfied, the infimum in question is equal to the maximum of $\sum_{i=1}^{m} \lambda_{i} \alpha_{i}$, when $\lambda_{i}$ vary under the conditions $\lambda_{i} \geqq 0$ and $\psi=\sum_{i=1}^{m} \lambda_{i} \phi_{i}$. Results analogous to this, but concerning a system of linear inequalities $\phi_{i}(x) \geqq \alpha_{i}$ [instead of the strict inequalities (6)] have been given by Gale, Kuhn and Tucker [5]. 
3. We proceed now to prove the theorems stated above.

Proof of THEOREM 1. That the two alternatives exclude each other is clear. Let $E^{m}$ denote the $m$-dimensional Euclidean space. Let $A$ denote the set in $E^{m}$ formed by all points of the form $\left(f_{1}(x), f_{2}(x), \cdots\right.$, $f_{m}(x)$ ), where $x \in K$. Let $B$ denote the convex hull of $A$. Denote by $N$ the open convex set in $E^{m}$ formed by all points with negative coordinates. Consider an arbitrary point $\left(\xi_{1}, \xi_{2}, \cdots, \xi_{m}\right)$ in $B$. There exist a finite number of elements $x_{1}, x_{2}, \cdots, x_{n}$ in $K$ and $n$ numbers $\alpha_{j} \geqq 0(1 \leqq j \leqq n)$ with $\sum_{j-1}^{n} \alpha_{j}=1$ such that $\xi_{i}=\sum_{j=1}^{n} \alpha_{j} f_{i}\left(x_{j}\right)$ $(1 \leqq i \leqq m)$. Since $f_{i}$ is convex, we have $\xi_{i} \geqq f_{i}\left(\sum_{j=1}^{n} \alpha_{j} x_{j}\right)$ for each $i$. Now assume that (1) is inconsistent. Then for at least one $i$, we have $f_{i}\left(\sum_{j=1}^{n} \alpha_{j} x_{j}\right) \geqq 0$ and therefore also $\xi_{i} \geqq 0$. In other words, the convex set $B$ is disjoint from the open convex set $N$. By the separation theorem of convex sets (see, e.g., [1, p. 71, Proposition 1 and p. 52, Proposition 16]), there exist $m$ numbers $\lambda_{i}$, not all zero, such that

$$
\sum_{i=1}^{m} \lambda_{i} \xi_{i}<0 \quad \text { for }\left(\xi_{1}, \xi_{2}, \cdots, \xi_{m}\right) \in N
$$

and

$$
\sum_{i=1}^{m} \lambda_{i} \xi_{i} \geqq 0 \quad \text { for }\left(\xi_{1}, \xi_{2}, \cdots, \xi_{m}\right) \in B .
$$

The first relation implies $\lambda_{i} \geqq 0(1 \leqq i \leqq m)$, the second relation implies (2).

Another Proof of Theorem 1. For each $x \in K$, let

$$
U(x)=\left\{\left(\xi_{1}, \xi_{2}, \cdots, \xi_{m}\right) \in E^{m} \mid \xi_{i}>f_{i}(x) \text { for } 1 \leqq i \leqq m\right\} .
$$

Let $U=\bigcup_{x \in K} U(x)$. Because the $f_{i}$ 's are convex, the open set $U$ is convex. Assume that (1) is inconsistent. Then $U$ does not contain the origin $0=(0,0, \cdots, 0)$. Therefore, again by the separation theorem of convex sets, there exist $m$ numbers $\lambda_{i}$, not all zero, such that $\sum_{i=1}^{m} \lambda_{i} \xi_{i}>0$ for all $\left(\xi_{1}, \xi_{2}, \cdots, \xi_{m}\right) \in U$. This implies (2) and $\lambda_{i} \geqq 0(1 \leqq i \leqq m)$. In fact, for any point $\left(\xi_{1}, \xi_{2}, \cdots, \xi_{m}\right) \in U$ and for any $\eta>0$, the point $\left(\xi_{1}, \cdots, \xi_{i-1}, \xi_{i}+\eta, \xi_{i+1}, \cdots, \xi_{m}\right)$ remains in $U$. If $\lambda_{i}<0$, then by taking $\eta>0$ sufficiently large, we would have $\lambda_{i}\left(\xi_{i}+\eta\right)+\sum_{j \neq i} \lambda_{j} \xi_{j}<0$.

Proof of Theorem 2. (i) We need only prove the "only if" part. Assume that (3) is a consequence of (1). Then the system obtained by adjoining the inequality $-g(x)<0$ to $(1)$ is inconsistent. Application of Theorem 1 to this inconsistent system yields the desired condition. 
(ii) $\gamma$ is finite, if and only if there exists a real number $\beta$ such that the inequality $g(x)-\beta \leqq 0$ is a consequence of (1). Then the condition for $\gamma$ to be finite follows from (i). Assume now that $\gamma$ is finite. Then $g(x)-\gamma \leqq 0$ is a consequence of (1). By (i), there are $\rho_{i} \geqq 0$ such that $g(x)-\sum_{i=1}^{m} \rho_{i} f_{i}(x) \leqq \gamma$ for all $x \in K$. On the other hand, for any $\epsilon>0$, there is a solution $x_{\epsilon} \in K$ of (1) such that $g\left(x_{\epsilon}\right)>\gamma-\epsilon$. If $\lambda_{i} \geqq 0$ and if $\alpha$ denotes the supremum of $g(x)-\sum_{i=1}^{m} \lambda_{i} f_{i}(x)$ over $K$, then $\gamma-\epsilon<g\left(x_{\epsilon}\right) \leqq \alpha+\sum_{i=1}^{m} \lambda_{i} f_{i}\left(x_{\epsilon}\right) \leqq \alpha$ for every $\epsilon>0$. Hence $\gamma \leqq \alpha$ and (5) is proved.

Proof of Theorem 3. First, by Theorem 2, (i), there are $m$ numbers $\lambda_{i} \geqq 0$ satisfying (4). As (3) is not a consequence of any proper subsystem of (1), we have $\lambda_{i}>0(1 \leqq i \leqq m)$. Suppose, if possible, that $\mu_{i}$ are real numbers, not all zero, such that $\sum_{i=1}^{m} \mu_{i} f_{i}(x) \geqq 0$ for all $x \in K$. Then as (1) is consistent, at least one of the $\mu_{i}$ 's is $<0$. Let $I=\left\{i \mid \mu_{i}<0\right\}$ and $\nu=\operatorname{Max}_{i \in I} \lambda_{i} / \mu_{i}<0$. Put $\eta_{i}=\lambda_{i}-\nu \mu_{i}(1 \leqq i \leqq m)$. Then

$$
g(x) \leqq \sum_{i=1}^{m} \lambda_{i} f_{i}(x) \leqq \sum_{i=1}^{m} \lambda_{i} f_{i}(x)-\nu \sum_{i=1}^{m} \mu_{i} f_{i}(x)=\sum_{i=1}^{m} \eta_{i} f_{i}(x)
$$

for all $x \in K$. But each $\eta_{i} \geqq 0$ and at least one of them is 0 , so by Theorem 2, (i), (3) would be a consequence of some proper subsystem of (1). This proves that the convex functions $f_{1}, f_{2}, \cdots, f_{m}$ are linearly independent.

Proof of Theorem 4. Assume that (1) is irreducibly inconsistent. Then (b) follows from Theorem 1 . The inequality $-f_{m}(x) \leqq 0$ is a consequence of the consistent system

$$
f_{i}(x)<0 \quad(1 \leqq i \leqq m-1)
$$

without being a consequence of any proper subsystem of (8). Hence by Theorem 3 , the $m-1$ convex functions $f_{1}, f_{2}, \cdots, f_{m-1}$ are linearly independent. This proves (a). Conversely, by Theorem 1, (a) implies that every proper subsystem of (1) is consistent, (b) implies the inconsistency of (1).

4. Instead of system (1) of strict inequalities, one may wish to consider the system

$$
f_{i}(x) \leqq 0,
$$

where $f_{i}$ are as before convex functions defined on a convex set $K$. From a known result on linear inequalities (see e.g., [3, Theorem 1]), the following conjecture would seem to be reasonable: If (9) is inconsistent, then there exist $m$ non-negative numbers $\lambda_{i}$ such that 
$\sum_{i=1}^{m} \lambda_{i} f_{i}(x)>0$ for all $x \in K$. This conjecture turns out to be false. Let $X$ be the Euclidean plane, where points are denoted by $x$ $=\left(\xi_{1}, \xi_{2}\right)$. Let $K$ be the convex set which is the union of the open halfplane $\xi_{2}>0$ and the half-line $\xi_{1}>0, \xi_{2}=0$. Let $f_{1}, f_{2}$ be defined on $K$ by $f_{1}(x)=\xi_{1}, f_{2}(x)=\xi_{2}$. Then the system $f_{i}(x) \leqq 0(i=1,2)$ is inconsistent on $K$, but no pair of non-negative numbers $\lambda_{1}, \lambda_{2}$ can satisfy $\lambda_{1} \xi_{1}$ $+\lambda_{2} \xi_{2}>0$ for all $\left(\xi_{1}, \xi_{2}\right) \in K$.

5. In this final section, we indicate that Theorems 1 and 2 can be extended to a much more general situation. Let $K$ be as before a nonempty convex set in a vector space $X$ over the real field. Let $Y$ be a locally convex topological real vector space. Let $P$ be a convex cone in $Y$ with nonempty interior. The interior of $P$ will be denoted by Int $P$. A function $f$ defined on $K$ and taking its values in $Y$ is said to be convex, if

$$
\alpha_{1} f\left(x_{1}\right)+\alpha_{2} f\left(x_{2}\right)-f\left(\alpha_{1} x_{1}+\alpha_{2} x_{2}\right) \in P
$$

holds for any two elements $x_{1}, x_{2} \in K$ and for any $\alpha_{1} \geqq 0, \alpha_{2} \geqq 0$, $\alpha_{1}+\alpha_{2}=1$. Then Theorems 1, 2 can be generalized, if we replace the system (1) of inequalities involving $m$ real-valued convex functions by a single relation $-f(x) \in \operatorname{Int} P$, where $f$ is a convex function defined on $K$ and taking its values in $Y$.

\section{REFERENCES}

1. N. Bourbaki, Espaces vectoriels topologiques, Chapters I, II, Paris, 1953.

2. W. B. Carver, Systems of linear inequalities, Ann. of Math. vol. 23 (1921-1922) pp. 212-220.

3. K. Fan, On systems of linear inequalities, Linear Inequalities and Related Systems (Ann. of Math. Studies, No. 38), edited by H. W. Kuhn and A. W. Tucker, Princeton, 1956, pp. 99-156.

4. J. Farkas, Theorie der einfachen Ungleichungen, J. Reine Angew. Math. vol. 124 (1902) pp. 1-27.

5. D. Gale, H. W. Kuhn and A. W. Tucker, Linear programming and the theory of games, Activity Analysis of Production and Allocation, edited by T. C. Koopmans, New York, 1951, pp. 317-329.

6. J. von Neumann and O. Morgenstern, Theory of games and economic behavior, Princeton, 1944.

7. H. Weyl, Elementare Theorie der konvexen Polyeder, Comment. Math. Helv. vol. 7 (1935) pp. 290-306.

The American University,

UNIVERSITY OF NOTRE DAME AND

National Bureau of Standards 